\title{
Face adaptation in an isolated population of African hunter-gatherers: Exposure influences perception of other-ethnicity faces more than own-ethnicity faces
}

\author{
Anthony C. Little ${ }^{1}$ Coren L. Apicella ${ }^{2}$
}

Published online: 18 August 2015

(C) Psychonomic Society, Inc. 2015

\begin{abstract}
Previous experiments have demonstrated that exposure to faces can change the perception of normality in new faces, such that faces similar to those at exposure appear more normal. Here we examined how experience influences adaptation effects in African Hadza hunter-gatherers, who have limited experience with White faces. We exposed participants to sets of either Hadza or White European faces that were manipulated to possess either wide-spaced or narrow-spaced eyes. We collected normality judgments both pre-exposure and post-exposure by showing pairs of images, one with wide-spaced and one with narrow-spaced eyes. Examining the difference between the preexposure and post-exposure judgments revealed that participants selected an increased number of images that were congruent with the faces to which they had been exposed. The change in normality judgments was strongest for White faces, suggesting that representations of White ethnicity faces are more malleable and less robust to adaptation, potentially because of the decreased experience that individuals had with them. A second experiment using the same test stimuli with a sample of White participants revealed equivalent adaptation effects for both Hadza and White faces. These data highlight the role of experience on the high-level visual adaptation of faces.
\end{abstract}

Keywords Aftereffects · Adaptation - Experience · Face processing $\cdot$ Exposure $\cdot$ Cross-cultural $\cdot$ Own race . Hunter-gatherers

Anthony C. Little

anthony.little@stir.ac.uk

1 School of Natural Sciences, University of Stirling, Stirling FK9 4LA, Scotland, UK

2 Department of Psychology, University of Pennsylvania, Philadelphia, PA 19104-6241, USA
For each class of stimuli that the human visual system encounters, it may develop an individual representation, or prototype, which is an average of the characteristics of all of the different stimuli that have been seen of that type (Apicella, Little, \& Marlowe, 2007; Giese \& Leopold, 2005; Loffler, Yourganov, Wilkinson, \& Wilson, 2005; Valentine, 1991). Much research regarding prototype formation has involved faces, and the notion of a multidimensional "face space" has proved extremely useful in helping to understand how faces are represented (Valentine, 1991). In the face space model, each individual face can be thought of as representing a different point in space, where the theoretical average of all faces occurs at the center. Individual faces lie on trajectories or vectors moving away from the central "average" face (Valentine, 1991).

Studies of face adaptation have been taken as evidence for a prototype model. Exposure to faces (i.e., adaptation) has been found to bias subsequent perceptions of novel faces by causing faces similar to those initially viewed to appear more prototypical, or normal, than they would otherwise be perceived, reflecting the recalibration of a prototype in light of recent visual experience (Leopold, O'Toole, Vetter, \& Blanz, 2001; Leopold, Rhodes, Muller, \& Jeffery, 2005; Rhodes, Jeffery, Watson, Clifford, \& Nakayama, 2003; Webster, Kaping, Mizokami, \& Duhamel, 2004; Webster \& MacLeod, 2011). For example, adaptation to faces with contracted features (i.e., internal facial features that are brought closer to the center of the face) causes individuals to perceive novel faces with contracted features as being more normal (Rhodes, et al., 2003). Analogous visual aftereffects using other facial stimuli, including faces that vary in identity (Leopold et al., 2001), ethnicity (Little, DeBruine, Jones, \& Waitt, 2008a; Webster et al., 2004), sex (Webster et al., 2004), expression (Webster et al., 2004), and sexual dimorphism (Buckingham et al., 2006; Little, DeBruine, \& Jones, 2005), have been observed. 
One previous study has examined the influence of familiarity on face adaptation. Using adaptation to distorted versions of the participant's own face, a famous face, or an unfamiliar face, smaller adaptation effects were observed when participants judged their own face than when they looked at other face types (Laurence \& Hole, 2011). This finding suggests that greater experience/familiarity with specific faces may create a robust representation that is more difficult to alter via adaptation. Here, we examined the effects of familiarity on different classes of facial stimuli, so that we could move beyond the role of familiarity with respect to specific individuals. Studies have demonstrated that the perception of race or ethnicity of faces may depend on separable representations (Apicella et al., 2007; Jaquet, Rhodes, \& Hayward, 2008; Little et al., 2008a), meaning that individuals could have more or less robust representations of different groups. Indeed, individuals have an advantage in recognizing members of their own race (e.g., Valentine, 1991). Poorer performance in recognition of other-race faces might be linked to stored representations, because experience with other-race faces can reduce the disadvantages in processing other-race faces (e.g., Sangrigoli, Pallier, Argenti, Ventureyra, \& de Schonen, 2005).

In Experiment 1, using a relatively isolated population of hunter-gatherers as our sample, we investigated whether adaptation effects were greater for a class of face stimuli that participants had more experience with (i.e., own-ethnicity faces) than for a class of stimuli with which they had limited experience (i.e., White faces). Specifically, we predicted that adaptation in Hadza hunter-gatherers would be greater for stimuli involving pictures of White Europeans than for pictures of African Hadza. In Experiment 2, we also examined adaptation effects in a White European sample. We predicted that White individuals would show either stronger adaptation to Hadza than to White faces, due to less experience with African faces, or more equivalent adaptation effects for both picture sets, due to having experience with African faces from living in a multiethnic culture and through media exposure.

\section{Experiment 1}

\section{Methods}

Participants The participants were 36 Hadza from remote regions of Northern Tanzania in Africa (19 men, 17 women; $18-64$ years of age, mean $=37.9, S D=12.2$ ). The Hadza are nomadic hunter-gatherers who live in a savannah-woodland habitat in Tanzania in camps that average about 30 individuals. The camps themselves continually shift location in response to ecological pressures. Camp residence is fluid and individuals choose their future campmates based on friendship and genetic relatedness. (Apicella, Marlowe, Fowler, \& Christakis, 2012). The participants were recruited by
Apicella from three separate camps in the Lake Eyasi region of Northern Tanzania and were interviewed in private in Swahili. All participants were fluent in Swahili.

Stimuli We used composite face stimuli made by combining the images of several men into a single image, which was then transformed to possess either wide or narrow-spaced eyes. All stimuli were constructed using established techniques (Little et al., 2005; Perrett et al., 1998) for manipulating the appearance of face images (for technical details, including mathematical algorithms, see Rowland \& Perrett, 1995; Tiddeman, Burt, \& Perrett, 2001). The face images were randomly selected from pre-existing databases of Hadza and White European male faces used in previous studies (Apicella et al., 2007; Apicella et al., 2012; Little et al., 2008b).

Composite images were made by delineating feature points on the faces, aligning the points in each image to the average of the images in an individual composite, and superimposing the images to create a photographic-quality image with the average shape and color of the constituent images (Rowland \& Perrett, 1995; Tiddeman et al., 2001). All of the images were standardized for size on interpupillary distance prior to transformation.

For use in the adaptation phase, ten composite images (each made from two images) from each ethnic group were transformed for eye spacing. This created wide and narrowspaced versions of each, making 40 images (ten White Europeans with wide eye spacing, ten White Europeans with narrow eye spacing, ten Hadza with wide eye spacing, and ten Hadza with narrow eye spacing).

For use in the pre-test and post-test, five different composite images (each made from random pairs of the adapting faces, four images each) from each ethnic set were also transformed for eye spacing. This created wide-spaced and narrowspaced versions, making ten pairs of images (five White Europeans with wide eye spacing vs. five White Europeans with narrow eye spacing, five Hadza with wide eye spacing vs. five Hadza with narrow eye spacing).

Eye spacing was manipulated by transforming the images relative to a pair of face images: one original image and one image in which all of the points delineating the eyes had been moved outward (Fig. 1). In other words, we used the shape difference between two images, which varied only in the distance between the eyes, to transform our images to have either a wider or a narrower eye spacing.

The distance change in eye spacing from the original (measured from the center of the eye) in the presented images was 21 pixels for each individual face, either wider or narrower (i.e., the original eye spacing equaled 0 ; narrow eye spacing equaled -21 pixels; and wide eye spacing equaled +21 pixels). The average face width was approximately 300 pixels. This meant a percentage change of eye spacing between the wide and narrow images of around $14.0 \%$ of the width of the faces. 


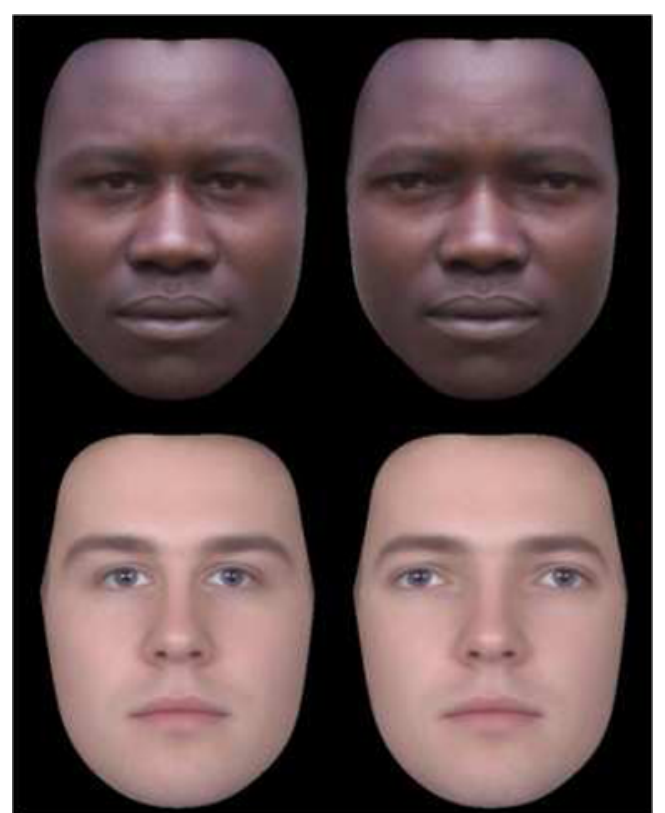

Fig. 1 Example European and Hadza face images (left, narrow eye spacing; right, wide eye spacing) from the pre-test and post-test

This procedure for manipulating eye spacing in face images has been used before (Little et al., 2005).

Procedure After age and sex were recorded, participants were orally presented with instructions: "In this study you will see faces and be asked to say which appears more normal. You will be asked to look at the pictures and choose the face twice." The experimenter then administered the forced choice pre-test manually. Image pairs were randomly selected, and image presentation on the left or the right side was also random. This was achieved via shuffling. Participants were asked "Which appears most normal?" for each pair, and after the experimenter had noted the answer, the next trial began. A total of five pairs were presented in the pre-test. After the pre-test, participants were exposed to either the wide-eyed or the narrow-eyed face set (the adaptation phase), determined randomly. Participants were continually told to "Look at each picture closely." Images were shown for $5 \mathrm{~s}$ (measured via stopwatch) and were presented in a random order. After the adaptation phase, participants completed the post-test which was identical to the pre-test.

\section{Results}

The percentages of wide-spaced eyes chosen pre-exposure and post-exposure were calculated for each participant. We then calculated a difference score reflecting the direction and strength of adaptation by subtracting the percentage of widespaced eyes chosen at pre-exposure from the post-exposure percentage (post-test minus pre-test score). Positive scores indicated that the choice of wide-spaced eyes as normal increased post-exposure, whereas negative scores indicated that the choice of wide-spaced eyes as normal decreased post-exposure.

A univariate analysis of covariance (ANCOVA) was conducted on strength-of-adaptation scores, with Exposure Condition (wide/narrow eyes) and Face Ethnicity (European/ Hadza) as between-participants factors and age entered as a covariate (because of the large age range of the sample). This revealed a significant main effect of exposure condition $[F(1$, $\left.31)=45.79, p<.001, \eta_{\mathrm{p}}{ }^{2}=.596\right]$ and a significant interaction between exposure condition and face ethnicity $[F(1,31)=$ $\left.4.43, p=.043, \eta_{\mathrm{p}}{ }^{2}=.125\right]$. No other effects or interactions were significant [all $\left.F_{\mathrm{s}}(1,31)<2.34, p \mathrm{~s}>.136, \eta_{\mathrm{p}}{ }^{2} \mathrm{~s}<.070\right]$. From Fig. 2, Hadza participants were influenced by exposure, such that those exposed to wide-spaced eyes came to choose more images with wide-spaced eyes; the converse was found for narrow-spaced eyes. This effect was stronger for participants exposed to White faces than for participants exposed to Hadza faces.

Removing age as a covariate from the model revealed an identical pattern of results, although for the interaction between exposure condition and face ethnicity the $p$ value increased slightly $\left[F\left(1,32=4.04, p=.053, \eta_{\mathrm{p}}{ }^{2}=.112\right]\right.$. Adding Sex of Participant to the model as a between-participants factor revealed no main effect and no interactions involving sex of participant [all $\left.F \mathrm{~s}(1,27)<0.22, p \mathrm{~s}>.642, \eta_{\mathrm{p}}{ }^{2} \mathrm{~s}<.008\right]$.

Independent-samples $t$ tests comparing strength-ofadaptation scores between exposure conditions revealed significant differences between scores in both those exposed to White faces $[t(14)=8.10, p<.001, d=4.32]$ and those exposed to Hadza faces $[t(18)=3.15, p=.006, d=1.48]$.

Additional post-hoc exploratory analyses were conducted to examine the influence of a participant's age on adaptation effect size. To do this, we reverse-coded the difference scores for participants in the narrow eye-spacing exposure condition. This produced a variable that reflected the strength of the adaptation effect, so that high scores indicated a greater impact of adaptation irrespective of condition. A custom-model ANCOVA was conducted on the direction-independent

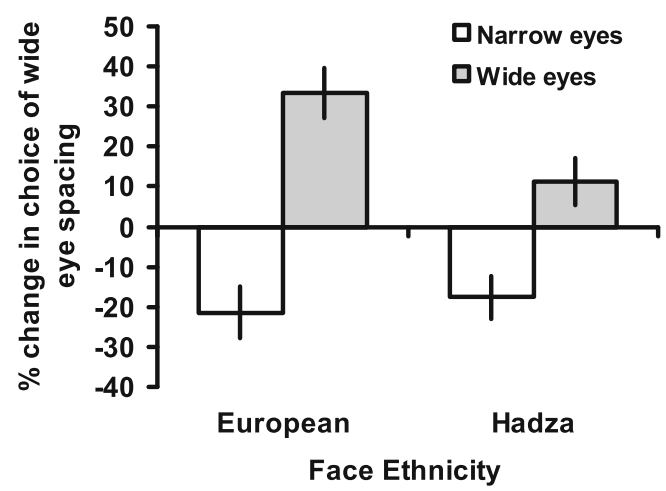

Fig. 2 Experiment 1: Percentage changes in choices of wide eye spacing $( \pm 1 S E$ of the mean) after exposure to wide-spaced or narrow-spaced eyes, split by ethnicity of exposure (European or Hadza) in Hadza participants 
strength-of-adaptation scores, with Face Ethnicity (European/ Hadza) as a between-participants factor and age entered as a covariate. In the custom model, we tested for a main effect of age and an interaction between face ethnicity and age. This revealed a significant interaction between age and face ethnicity $\left[F(1,33)=6.30, p=.017, \eta_{\mathrm{p}}{ }^{2}=.160\right]$ and no main effect of age $\left[F(1,33)=0.34, p=.562, \eta_{\mathrm{p}}{ }^{2}=.010\right]$.

To parse the interaction, the sample was split by face ethnicity and Pearson product-moment correlations were computed between age and the adaptation effects. A positive correlation between age and adaptation effect for European faces $(r=.368, p=.161)$ and a negative correlation between age and adaptation effect for Hadza faces $(r=-.396, p=.084)$ were found. Although neither correlation was significant, they were significantly different from each other $(Z=2.18, p=.029$; see also the significant interaction above). Older participants were influenced more by exposure to European faces when judging the normality of European faces and were influenced less by exposure to Hadza faces when judging Hadza faces.

\section{Experiment 2}

In Experiment 1, Hadza individuals demonstrated stronger adaptation effects with White faces than with Hadza faces. As a follow-up experiment, we examined adaptation effects using the same stimuli in a White population.

\section{Method}

Participants The participants were 89 self-reporting White individuals (23 men, 66 women; 16-68 years of age, mean = $30.0, S D=11.9)$. Participants were recruited over the Internet and tested online. They were selected for reporting to be of White ethnicity. The participants were of various nationalities: British $(N=39)$, other European $(N=21)$, North American $(N=16)$, South American $(N=9)$, Other $(N=2)$, African $(N=1)$, and Australian $(N=1)$.

Stimuli The stimuli were the same as in Experiment 1.

Procedure The experiment was administered online via a computer screen. Participants filled in a short questionnaire asking for their age and sex. The remaining procedures were identical to those of Experiment 1, except that the instructions were read in English rather than verbally stated in Swahili, and randomization of the picture sets and presentation order were computed via computer program rather than via shuffling.

\section{Results}

A strength-of-adaptation score was calculated for each participant, as in Experiment 1.
A univariate ANCOVA was conducted on the strength-ofadaptation scores, with Exposure Condition (wide/narrow eyes) and Face Ethnicity (European/Hadza) as betweenparticipants factors and age entered as a covariate. This revealed a significant main effect of exposure condition $[F(1$, $\left.84)=38.54, p<.001, \eta_{\mathrm{p}}{ }^{2}=.315\right]$ and no significant interaction between exposure condition and face ethnicity $[F(1,84)=$ $\left.0.07, p=.791, \eta_{\mathrm{p}}{ }^{2}=.001\right]$. We observed a close-to-significant effect of face ethnicity $\left[F(1,84)=3.52, p=.064, \eta_{\mathrm{p}}{ }^{2}=.040\right]$, which reflected that, irrespective of condition, wide-spaced eyes were seen as more normal in White faces than in African faces after exposure. There was no main effect of age $\left[F(1,84)=0.36, p=.552, \eta_{\mathrm{p}}^{2}=.004\right]$. From Fig. 3, White participants were influenced by exposure, such that those exposed to wide-spaced eyes came to choose widespaced eyes as normal more often; the converse was found for narrow-spaced eyes. Participants exposed to White and Hadza faces showed similar effects.

Removing age as a covariate from the model revealed an identical pattern of results. Adding Sex of Participant to the model as a between-participants factor revealed no main effect and no interactions involving sex of participant $[$ all $F \mathrm{~s}(1,80)<$ 2.55, $\left.p \mathrm{~s}>.114, \eta_{\mathrm{p}}{ }^{2} \mathrm{~s}<.031\right]$.

Independent-samples $t$ tests comparing strength-ofadaptation scores between exposure conditions revealed significant effects of condition for both those exposed to White $[t(40)=4.27, p<.001, d=1.35]$ and those exposed to Hadza $[t(45)=4.65, p<.001, d=1.39]$ faces.

As in Experiment 1, we calculated a variable that reflected the strength of the adaptation effect, in which high scores indicated a greater impact of adaptation irrespective of condition. A custom-model ANCOVA was conducted on the adaptation effect, with Face Ethnicity (European/Hadza) as a between-participants factor and age entered as a covariate. In the custom model, we tested for a main effect of age and an interaction between face ethnicity and age. This revealed no significant interaction between age and face ethnicity $[F(1$, $\left.86)=0.02, p=.883, \eta_{\mathrm{p}}{ }^{2}<.001\right]$ and no main effect of age

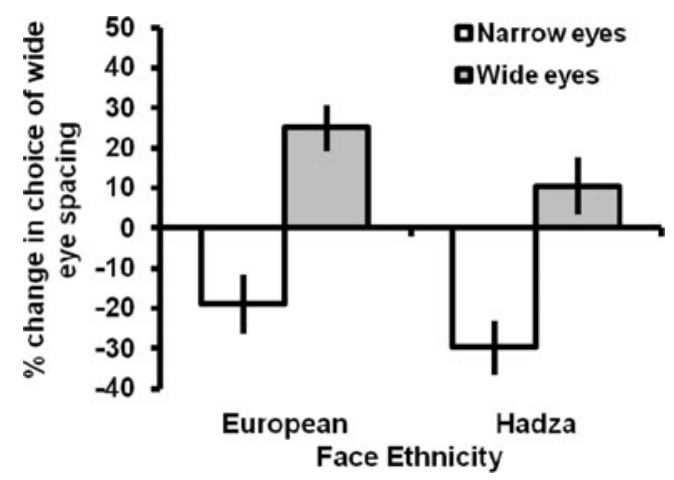

Fig. 3 Experiment 2: Percentage changes in choices of wide eye spacing $( \pm 1 S E$ of the mean) after exposure to wide-spaced or narrow-spaced eyes, split by ethnicity of exposure (European or Hadza) in White participants 
$\left[F(1,86)=0.13, p=.717, \eta_{\mathrm{p}}^{2}=.002\right]$. Age did not significantly affect the strength of adaptation in the White sample.

\section{Discussion}

Prior research suggests that exposure to faces biases subsequent perceptions of novel faces (Bestelmeyer et al., 2008; Buckingham et al., 2006; Leopold et al., 2001; Leopold et al., 2005; Little et al., 2005; Little et al., 2008a; Rhodes et al., 2003; Webster et al., 2004; Webster \& MacLeod, 2011), and we found similar effects in a relatively isolated population of hunter-gatherers. Of greater interest, Hadza participants also demonstrated greater effects of adaptation for White faces, with which they have limited experience, than for Hadza faces. In contrast, White participants demonstrated equivalent adaptation effects for White and African faces.

Adaptation effects have been shown to be smaller when judging one's own face rather than famous or unfamiliar faces (Laurence \& Hole, 2011), suggesting that greater experience results in more robust representations that are less prone to adaptation. Our study provided an important opportunity to examine the influence of experience on adaptation effects using different classes of faces. Since the Hadza have relatively little experience with White faces, they are an ideal population for this type of research, as compared to samples drawn from populations with access to modern media and who live and work in ethnically diverse places, such as in cities and universities (Apicella, 2011; Henrich, Heine, \& Norenzayan, 2010). For Hadza participants, we found that change in normality judgments in participants was stronger for White than for Hadza face stimuli. This finding highlights the role of familiarity and experience on the robustness of face representations. Our data are also consistent with the notion that individuals build distinct representations of faces based on all of the faces they have seen of a given class (Apicella et al., 2007; Jaquet et al., 2008; Little et al., 2008a) - in this instance, a representation of Hadza faces and a representation of White faces. If all faces encountered were compared to the same representation and influenced this representation independent of ethnicity, we would expect equivalent effects of adaptation for White and Hadza faces. The different effect sizes reported here (where the effect size for Hadza exposed to White faces was nearly three times the other effects) indicate that the ethnicity of faces matters to the size of the adaptation effect. Experience may then shape the representations of different classes of faces, and differential experience can render these representations more or less robust.

Post-hoc exploratory analyses for the Hadza demonstrated a significant interaction between participants' age and face ethnicity on the strength of the adaptation effect. Although we note our small sample size and nonsignificant correlations, these data are consistent with the notion that increased experience leads to more robust representations of faces of different ethnicities. If younger individuals have greater experience with White Europeans, then we can predict that their representations of these faces should be more robust and that adaptation effects would be weaker. Future studies can examine the roles of age and ethnicity on adaptation in other populations using larger samples.

In contrast to our findings with Hadza participants, we found no effect of face ethnicity on the strength of adaptation effects in our sample of White participants. It is possible that familiarity effects could have been found in this sample; however, we assumed that the White participants in our sample had received more exposure to faces of various ethnicities by way of living in more multiracial environments and by being exposed to media containing various ethnicities. The lack of a difference seen in the present study is suggestive that this exposure was sufficient to generate equivalently robust representations of White and African faces, at least for our adult sample here. We note, however, the relatively small sample size in our study and that experience with faces of other races has measurable impacts in domains such as recognition (e.g., Sangrigoli et al., 2005; Valentine, 1991). We additionally observed no effect of or interaction with age in the White sample, suggesting that younger and older participants also possessed equivalently robust representations of White and African faces.

In summary, in a sample of African hunter-gatherers with relatively limited experience of White faces, adaptation effects appeared strongest for faces with which individuals had more limited experience. These data highlight the important role of experience in generating robust representations of different classes of faces.

\section{References}

Apicella, C. L. (2011). On the universality of attractiveness. In M. Brockman (Ed.), Future science: Essays from the leading edge (pp. 89-100). New York: Vintage.

Apicella, C. L., Little, A. C., \& Marlowe, F. W. (2007). Facial averageness and attractiveness in an isolated population of hunter-gatherers. Perception, 36, 1813-1820.

Apicella, C. L., Marlowe, F. W., Fowler, J. H., \& Christakis, N. A. (2012) Social networks and cooperation in hunter-gatherers. Nature, 481, 497-501. doi:10.1038/nature10736

Bestelmeyer, P. E. G., Jones, B. C., DeBruine, L. M., Little, A. C., Perrett, D. I., Schneider, A., . . Conway, C. A. (2008). Sex-contingent face aftereffects depend on perceptual category rather than structural encoding. Cognition, 107, 353-365. doi:10.1016/j.cognition.2007. 07.018

Buckingham, G., DeBruine, L. M., Little, A. C., Welling, L. L. M., Conway, C. A., Tiddeman, B. P., \& Jones, B. C. (2006). Visual adaptation to masculine and feminine faces influences generalized preferences and perceptions of trustworthiness. Evolution and Human Behavior, 27, 381-389. 
Giese, M. A., \& Leopold, D. A. (2005). Physiologically inspired neural model for the encoding of face spaces. Neurocomputing, 65, 93-101.

Henrich, J., Heine, S. J., \& Norenzayan, A. (2010). The weirdest people in the world? Behavioral and Brain Sciences, 33, 61-83, disc. 83135. doi:10.1017/S0140525X0999152X

Jaquet, E., Rhodes, G., \& Hayward, W. G. (2008). Race-contingent aftereffects suggest distinct perceptual norms for different race faces. Visual Cognition, 16, 734-753.

Laurence, S., \& Hole, G. (2011). The effect of familiarity on face adaptation. Perception, 40, 450-463.

Leopold, D. A., O’Toole, A. J., Vetter, T., \& Blanz, V. (2001). Prototypereferenced shape encoding revealed by high-level aftereffects. Nature Neuroscience, 4, 89-94. doi:10.1038/82947

Leopold, D. A., Rhodes, G., Muller, K. M., \& Jeffery, L. (2005). The dynamics of visual adaptation to faces. Proceedings of the Royal Society B, 272, 897-904.

Little, A. C., DeBruine, L. M., \& Jones, B. C. (2005). Sex-contingent face after-effects suggest distinct neural populations code male and female faces. Proceedings of the Royal Society B, 272, 2283-2287.

Little, A. C., DeBruine, L. M., Jones, B. C., \& Waitt, C. (2008a). Category contingent aftereffects for faces of different races, ages and species. Cognition, 106, 1537-1547. doi:10.1016/j.cognition. 2007.06.008

Little, A. C., Jones, B. C., Waitt, C., Tiddeman, B. P., Feinberg, D. R., Perrett, D. I., . . Marlowe, F. W. (2008b). Symmetry is related to sexual dimorphism in faces: Data across culture and species. PLoS ONE, 3, e2106. doi:10.1371/journal.pone.0002106
Loffler, G., Yourganov, G., Wilkinson, F., \& Wilson, H. R. (2005). fMRI evidence for the neural representation of faces. Nature Neuroscience, 8, 1386-1390.

Perrett, D. I., Lee, K. J., Penton-Voak, I., Rowland, D., Yoshikawa, S., Burt, D. M., . . A Akamatsu, S. (1998). Effects of sexual dimorphism on facial attractiveness. Nature, 394, 884-887. doi:10.1038/29772

Rhodes, G., Jeffery, L., Watson, T. L., Clifford, C. W. G., \& Nakayama, K. (2003). Fitting the mind to the world: Face adaptation and attractiveness aftereffects. Psychological Science, 14, 558-566.

Rowland, D. A., \& Perrett, D. I. (1995). Manipulating facial appearance through shape and color. IEEE Computer Graphics and Applications, 15, 70-76.

Sangrigoli, S., Pallier, C., Argenti, A. M., Ventureyra, V. A. G., \& de Schonen, S. (2005). Reversibility of the other-race effect in face recognition during childhood. Psychological Science, 16, 440-444.

Tiddeman, B. P., Burt, D. M., \& Perrett, D. I. (2001). Prototyping and transforming facial texture for perception research. IEEE Computer Graphics and Applications, 21, 42-50.

Valentine, T. (1991). A unified account of the effects of distinctiveness, inversion, and race in face recognition. Quarterly Journal of Experimental Psychology, 43, 161-204.

Webster, M. A., \& MacLeod, D. I. A. (2011). Visual adaptation and face perception. Philosophical Transactions of the Royal Society B, 366, 1702-1725. doi:10.1098/rstb.2010.0360

Webster, M. A., Kaping, D., Mizokami, Y., \& Duhamel, P. (2004). Adaptation to natural facial categories. Nature, 428, 557-561. 